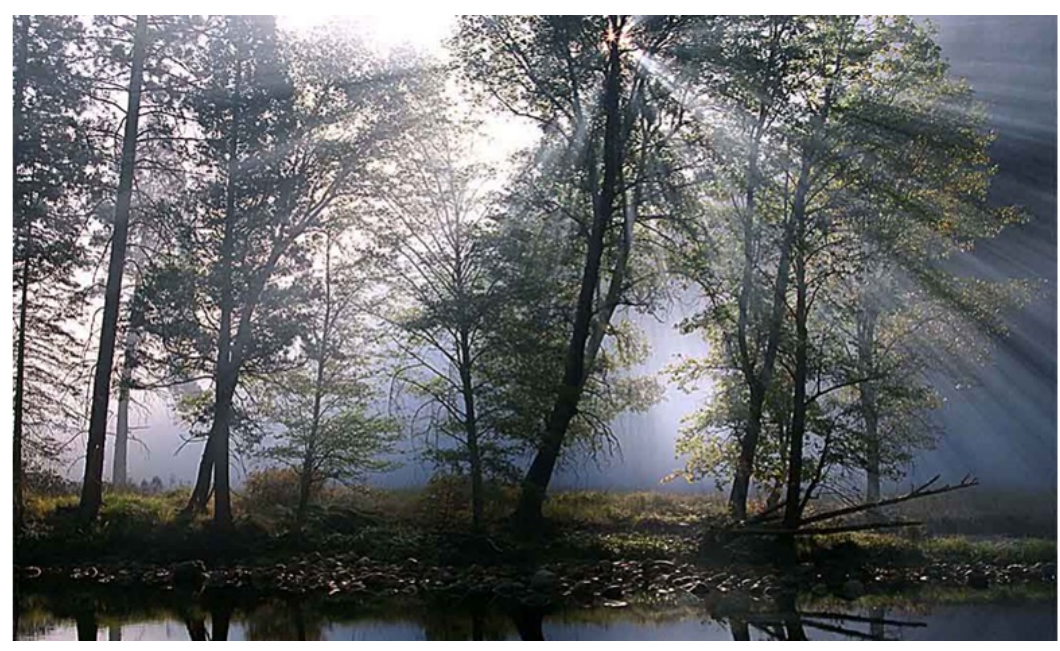

\title{
Extraterrestrial Extrapolations of Earthly Organismality
}

\author{
Tim Dumonceaux ${ }^{1}$
}

1 Agriculture and Agri-Food Canada

Funding: The author(s) received no specific funding for this work.

Potential competing interests: The author(s) declared that no potential competing interests exist.

\section{Abstract}

It has long been known that the microbial component of terrestrial ecosystems provides the capacity for nutrient and elemental cycling and other key ecosystem services. Advances in DNA sequencing technology and analytical capacity continue to reveal novel insights into the structure and dynamics of these microbial ecosystems. These insights have led to an increasing appreciation of the importance of the microbial species that associate with multicellular organisms, including plants. The relationship between the multicellular host and its microbiota is sufficiently interdependent that the plant has come to be understood as a "holobiont" that encompasses a multitude of prokaryotic and eukaryotic species. This expands the traditional view of what constitutes an independent organism to include a collectivity of species that coexist for mutual benefit. The concept of organismality presented by Queller and Strassman goes even further than that, arguing that organisms can be defined as collectivities of cells or species that share a common purpose, regardless of genetic identity. As applied to plants, the ideas elaborated by Simard and others that plant communities and their associated mycorrhizal networks form an interconnected collectivity with cognitive functions also fits into this theme. Stephen Jay Gould discussed ideas of what constitutes an organism in the 1990s. But the ideas themselves are even older than that, dating all the way back to Charles Darwin himself. Incredibly, the connections among these 
philosophical concepts were anticipated in a science fiction story written by Isaac Asimov over 70 years ago.

Good science fiction takes presently known scientific concepts and technologies and plausibly extrapolates them for the benefit of the story and the delight of the reader. Great science fiction does that, to be sure, but also has the quality of anticipating technological advances or, more importantly, changes in our ways of understanding the natural world. An example of the latter is the short story "Green Patches" by the great biochemist and science fiction writer Isaac Asimov, which was published in November, 1950 by Galaxy Science Fiction (the story was initially published with the editorially mandated title "Misbegotten Missionary". In the anthology in which I first encountered this story, Asimov derisively notes the annoying habit his editors had of changing the titles of his stories, and that "Green Patches" was his original and intended title). The story describes a planet initially explored by a scientist called Saybrook, who had first excitedly sent back reports of an Earthlike planet with a lush biosphere, then suddenly and mysteriously vaporized himself along with his ship and crew. A crew is sent from Earth to explore Saybrook's eponymous planet to determine the reason for this unexpected outcome of the initial mission. While Saybrook's Planet looked to have a very Earth-like biosphere, closer inspection revealed some important differences. In fact, life on Saybrook's Planet was bereft of some important features of the biosphere with which we are familiar:

“...there was no conflict for food on the planet. All plants grew pulpy appendages which were eaten by the animals. These were regrown in a matter of hours. No other parts of the plant were touched. It was as though the plants fed the animals as part of the order of nature...insects kept their numbers reasonable, though no birds ate them; the rodent-like things did not swarm, though no carnivores existed to keep them in check."

Furthermore, Asimov gives his biosphere a communal consciousness, and repeatedly flips the perspective in the story from the humans to the Saybrook life form. The "misbegotten missionary" of the story's altered title intended to board the ship, return to Earth, and transform the earthly biosphere into a unified, Saybrook-like organism. Unbeknownst to the human explorers, and despite their extreme precautions, part of the Saybrook biosphere gains access to the ship, a necessary first step in its mission to colonize and reform life on Earth. It was satisfied with its initial success, but quickly came to regret its more Earth-like situation in being parted from its own biosphere:

"And the thought faded out of satisfaction and into loneliness. It was a terribly unhappy and unnatural thing to be parted from all the rest of the unified organism, to be a life fragment oneself. How could these aliens stand being fragments?" 
Asimov's story, written over 70 years ago, describes a biosphere that works very differently from the only known one, that of our own Earth. All of the individual units of life on Saybrook's Planet were part of one single, unified organism. Life on our planet, we know very well, does not work through such communally beneficial cooperation among creatures of different species - our "life fragments" exist and change through a process of natural selection ("Nature red in tooth and claw" as described by Alfred, Lord Tennyson in a poem published a decade before Darwin transformed our understanding of biology). Clearly, our own biosphere is composed of innumerable life units that exist to survive, compete, reproduce, and adapt in a harsh, dangerous world with dynamic external conditions. Stephen Jay Gould noted [1] that “natural selection works upon individuals engaged in a struggle for reproductive success...Fine, but how shall we define the "individual" engaged in such a struggle?". Gould goes on to define an "evolutionary individual" (EI), or "unit of selection" as an entity that possesses five distinct properties: A clear beginning (i.e., birth); a clear ending (death); sufficient stability during its existence to be recognized as a distinct entity, which requires cooperation among its constituent units (for example, in multicellular animals, cells, tissues, organs, and systems); it must have the ability to bear offspring; and those offspring must resemble their parents, but with the possibility of some differences. It is upon these differences, of course, that natural selection works to adapt earthly life fragments to their environment over long periods of time.

So how (on Earth) do we define an organism anyway? According to Gould, "canonical individuals are bounded entities with a definable form - a whale, a tree, a cockroach, a human being". We generally think of an organism in this way, with the additional, more modern requirement that an organism should feature genetic identity (or near identity) within its constituent units (cells). Botanists, however, have developed concepts that expand upon this notion. For botanists, a "ramet" is the morphologically defined aspect of individuality from a clonal origin - Gould's canonical individual. Many plants, however, consist of a physically connected system of genetically identical ramets which appear, above ground, to be a collection of individuals. Thus we must also define a "genet", which is an entire clonal system of ramets. It is not only plants that feature this distinction between ramets and genets. Interconnected mats of the facultatively saprophytic white-rot Basidiomycete Armillaria are, by some measures, among the largest collections of ramets (i.e., genets) known, measuring up to square kilometres in size. Some animal genets are not even physically connected. For example, some female aphids reproduce by parthenogenesis, producing hundreds of clonal offspring whose genetic identity helps protect the mother aphid from predation, by sheer force of numbers (while any given aphid might be consumed, it is unlikely that all of the unconnected ramets will be, which increases the chances that the genet will be available for sexual reproduction and so subject to evolutionary processes). These considerations raise the question about whether an El can exist at other levels of organization - cannot genes, as "parts" of a genet, or species, as "collectivities" of genets, be equally valid evolutionary individuals?

Of course, there is an increasing appreciation that there are, indeed, earthly echoes of Saybrook's Planet. Research into the manifold associations between multicellular plant or animal hosts and their abundant and diverse microbiota have led to the concept of the "holobiont", which encompasses the 
canonical individual and its associated microbiota. Lyu et al. [2] discuss the concept of the plant holobiont as a collective individual comprised of cells from across the prokaryotic and eukaryotic kingdoms, with a broad range of associations represented. The plant-microbiome interaction ranges in intimacy from epiphytic (associated with the outside of the plant surface) to endophytic (bacteria and fungi existing within and between plant cells), all the way to the ancient association of microbes within incipient plant cells that ultimately became endosymbiotic, yielding cellular organelles (mitochondria and chloroplasts). They argue that all of these associations are sufficiently important to the functioning of the plant that without its associated microbes, the concept of the "plant" is incomplete. Through this lens, we can see that evolutionary individuals can be genetically highly disparate, including both plant (or animal) and microbial cells in a single system that works cooperatively together and competes with other individuals for reproductive success.

It is becoming understood that this view of the individual organism, a true collectivity that spans all of the domains of life (Eukarya, Bacteria, Archaea), accurately describes the associations and interdependencies that are present in the El. Such collectivities are fundamental to life in our biosphere, yet it is still a conceptually small step away from the traditional notion of the evolutionary individual interacting in a competitive and often destructive manner with other Els for reproductive success. The concept of expanded collectivities, consisting of interconnected holobionts that interact synergistically for the benefit both of the individuals and of the broader ecosystem, requires an intellectual leap towards the idea of the unified, noncompetitive biosphere envisioned by Asimov. Suzanne Simard of the University of British Columbia describes just such a network of holobionts in the forest and riparian ecosystems of British Columbia, Canada [3]. Simard discusses the notion that mycorrhizal networks connecting trees in the forest impart aspects of cognition - communication, behaviour, learning, and memory - onto the ecosystem itself. The network of interactions spans biological Kingdoms (plants, fungi, bacteria) and results in a system of connected holobionts that possess cognitive capabilities far beyond what has previously been associated with plants.

But how can a network of plants, which obviously lack a brain, possess cognitive functions? This paradox can be resolved by considering the notion that plants do, in fact, have brains, and that their brains are below ground - their root systems. The notion of the root system as the plant's brain is hardly new, having been first described nearly a century and a half ago by Charles Darwin himself, along with his son Francis. Their "root-brain" hypothesis, largely ignored at the time, has seen a reconsideration recently, as described by Baluška et al [4]. As quoted therein, the Darwins describe the root system as the plant's sensory organ:

"It is hardly an exaggeration to say that the tip of the radicle thus endowed [with sensitivity] and having the power of directing the movements of the adjoining parts, acts like the brain of one of the lower animals; the brain being seated within the anterior end of the body, receiving impressions from the sense-organs, and directing the several movements." 
It is important to note the use of the word, "anterior" to describe the location of the root system within the plant. Descriptions of plant growth and symmetry typically refer to the soil horizon - the shoot and root apical meristems both grow in a direction that is generally away from the soil (shoot going up; root going down). In animals such as dogs or fishes, the dorsal-ventral axis divides the animal into anterior (towards the head or front) and posterior (towards the back) ends. The anterior end contains the sensory organs (often with visual, olfactory, and auditory capabilities) and brain, while the posterior end contains organs for waste excretion and sexual reproduction. If we mentally turn the plant on its side, with the soil horizon becoming the "dorsal-ventral axis", and use the Darwins' view of the root system being seated at the anterior end of the organism, we can see that the "posterior" end of the plant (that which we see above ground) indeed contains the functions of waste exchange (i.e., stomata that expel oxygen and water vapour) and sexual reproduction - the floral organs. By analogy, then, the anterior end contains the plant's sensory and cognitive functions. Baluška et al. describe three "zones" within the root system with neuronal-like functionality: the tip of the root (meristem) contains strong mitotic activity; the transition zone behind it possesses synaptic activity; and the distal zone has cell elongation activity. They describe a directional cell-to-cell transfer of the plant growth hormone indole acetic acid (IAA; auxin) through the synaptic transition zone towards the apical mitotic zone where the compound has its effect on cell division. This directional transfer of the chemical along cells is analogous to neurotransmission in the animal brain in fact, Simard and others have pointed out that the chemical structure of IAA is very similar to that of serotonin, an important neurotransmitter. Could this be an example of convergent evolution? Moreover, Simard noted that plants invest a great deal - by some measures up to $90 \%$ - of their photosynthetic output below ground, suggesting that the root system is analogous to the glucose-hungry animal brain. Thus there are many parallels, at least conceptually, between plant root systems and animal brains.

Unlike animal brains, however, plant root systems are strongly interconnected with their microbiota. More than $95 \%$ of plant families feature interactions with mycorrhizal fungi, which are generally divided into ectomycorrhizae and arbuscular mycorrhizae. The ectomycorrhizal fungi (EMF, from the phyla Basidiomycota and Ascomycota) are mainly associated with gymnosperm trees, while the arbuscular mycorrhizal fungi (predominantly Glomeromycota) are associated with angiosperm trees and grasses in the forest biome. In both cases, the plant benefits from the fungal root associations because hyphal growth through the soil is less energetically expensive than root growth; the fungus exchanges the nutrients its hyphae obtain from the soil for photosynthate from the plant. There is a complex, coevolved system of communication between plant and fungal symbiont using bidirectional elicitor signal molecules such as IAA. Furthermore, Simard notes that the trees in the BC forest feature complex interconnectivities mediated through their mycorrhizal networks. In some of her early experiments, Simard fed young, established Douglas fir seedlings radiolabelled $\mathrm{CO}_{2}$ and was able to trace the radioactivity, within hours, to nearby plants that had not been exposed to the radioactive gas [5]. It became clear that the interconnected mycorrhizal networks very quickly move chemical signals between connected nodes in this 
forest ecosystem. The chemical signals exchanged among plants through the mycorrhizae go far beyond photosynthate, and include fixed nitrogen, water, defense molecules, kin recognition signals, allelochemicals, nutrient analogues, fungal carbohydrates, amino acids, phosphates, fungal nuclei, and, of course, the plant "neurotransmitter", IAA. This, according to Simard, constitutes communication in its truest sense - she notes that the word itself derives from the Latin communicat, meaning "to share". The chemical signals exchanged among the individual plants in the forest ecosystem constitute a language, with the individual chemicals themselves being the vocabulary of that language. This communication allows groups of plants to adapt to their environment, facilitated by their microbiota. In fact, Simard and her colleagues have mapped the complex network topology of evergreen conifers through their shared colonization with the EMF Rhizopognon vesiculosus, which identified older, highly interconnected "mother" trees that play a crucial role in the health of the forest ecosystem [6].

The mycorrhizal networks connecting plants give them true cognitive capabilities including behaviour, learning, and memory. Chemical transmission can affect behaviours by eliciting changes in plant morphology including rooting depth and growth height, and plant physiology such as photosynthetic activity and nutrient uptake. Simard notes that the survival of Douglas fir seedlings is improved when they can access the mycorrhizal network of older, established trees (the "mother tree" concept). Learning, another key aspect of cognition, is also facilitated by the root-associated mycorrhizae. Root exudates carry information that allow trees to recognize the genetic relatedness of other individuals within the network, and can allocate photosynthetic resources accordingly (facilitating the survival of offspring). In another example mediated by AMF, broad beans respond to aphid attack by sending defense signals to neighbouring plants through their mycorrhizal networks, so that the recipient plants learn of an imminent attack and can prepare their defense response [7]. These communicative interactions suggest a universally benevolent purpose wherein plants invest resources altruistically for the benefit of other individuals, but this is not always the case. Some of the allelochemicals that are shared through mycorrhizal networks restrict the growth of potentially competing holobiont neighbors [7], indicating that these interactions are, as might be expected in an advanced society, marvelously complex.

As for memory, Simard eloquently describes a pathway for ecosystem memory involving a wide variety of individual plants, animals, and microbiota. To illustrate this, she considers salmon eggs hatching in streams inland. The young fry then swim out to sea, where they spend much of their life obtaining nutrients from the ocean and incorporating these nutrients into their bodies. They then return to the mother streams to breed and continue the cycle, then die. The bounty represented by this return is not lost on the local animals (including humans, bears, and others), which gather the fish. Bears, for example, capture the fish and consume them in the forest and riverbanks, discarding the carcasses on the forest floor and depositing more of the fish-derived nutrients through their excrement. The EMF ultimately obtain these salmon nutrients through decomposition by the forest soil microbiota, and these nutrients satisfy much of the trees' nutritional requirements. Once so metabolized, the nutrients from the salmon, initially obtained in the ocean, are deposited in the tree rings for up to many centuries. This constitutes a 
"memory" of salmon runs, and the benefit of these interactions is reflected in faster tree growth along the banks of salmon streams and the moderation of water nutrient content and temperature by the trees themselves, which shapes the diversity of composition of the entire ecosystem. Thus, according to Simard, the salmon, carnivores, microbiota, and trees are a complex interconnected ecosystem with cognition, learning, and memory functions. Can this pan-domain interdependency be considered to be something like a Saybrook "organism"?

To understand this, we must reconsider the notion of organismality itself. Queller and Strassman [8] have argued that, rather than genetic identity, it is the notion of shared purpose that is the defining feature of an organism. According to this concept, an organism is a collectivity that works together for the benefit of the whole, featuring high levels of cooperation and low levels of conflict. West and Kiers [9] elaborate upon this notion of a two-variable concept of organismality that divides the properties of cooperation and conflict into four quadrants (see Figure 1 in West and Kiers). Applied to groups of cells, the four quadrants are defined as organisms, featuring high cooperation and low conflict (canonical organisms and holobionts fit into this category, but with different levels of the two variables); societies (e.g. biofilms), featuring high cooperation and high conflict; simple groups (e.g., yeast flocs), with low conflict and low cooperation; and competitors, which display low cooperation and high conflict. Applying this same concept to groups of multicellular individuals allows us to define a novel view of organismality that encompasses the ideas expressed by Simard and others. Using this framework, we can see that our aphid clones, despite their genetic identity, are more of a simple group than a single organism. Similarly, a collection of bamboo ramets, while interconnected and genetically identical, do not truly meet the definition of high cooperativity and low conflict that defines an organism. However, a wide array of groupings can, in this 2variable concept of organismality, be reasonably considered to be organisms, including the multi-Kingdom groups such as the Douglas fir-Rhizopognon interconnected groups in BC forests - as groups of genetically disparate individuals with high cooperation and low conflict, these are organisms in the truest sense.

We can then consider Asimov's Saybrook consciousness, conceived decades before any of these ideas were elaborated, as an extreme example of this concept of organismality, with very high levels of cooperation and very low levels of conflict - an extraterrestrial extrapolation of the earthly concept of organismality. As scientists, we are well aware of the dangers of extrapolation in reaching conclusions about natural phenomena, but such stretches of the imagination are exactly the arena of good science fiction. Our biosphere as a whole does not reach these levels of cooperation/conflict, but aspects of it certainly do.

I leave the final words to Stephen Jay Gould [1], a scientific and literary giant whose 30-year series of essays on the natural world served as an inspiration for generations of scientists. Gould commonly ended his essays with quotes that supported his central thesis, so it is fitting that he should supply a quote here. Gould states (and I could not agree more):

"Nature is not an intrinsic harmony of clearly defined units. Nature exits in multiple levels, 
interacting with fuzziness at their borders. We cannot even formulate an unambiguous definition of "individual" at the single level of organic bodies - as Armillaria mats and aphid clones demonstrate. Furthermore, in Darwinian terms, legitimate individuals exist at several levels of genealogical hierarchy - genes and species, as well as individuals. But what a fascination when this maelstrom of differing individuals builds its network of interaction to produce life's history by Darwinian evolution!"

Acknowledgments: I thank Janet Hill and Monika Gorzelak for commentary on this manuscript and stimulating discussions.

Conflicts of Interest: The author declares no conflict of interest.

\section{References}

1. Gould, S.J., A Humongous Fungus Among Us, in Dinosaur in a Haystack. 1995, Random House: New York. p. 335-343.

2. Lyu, D., J. Zajonc, A. Pagé, C.A.S. Tanney, A. Shah, N. Monjezi, L.A. Msimbira, M. Antar, M. Nazari, R. Backer, and D.L. Smith, Plant Holobiont Theory: The Phytomicrobiome Plays a Central Role in Evolution and Success. Microorganisms, 2021. 9(4): p. 675.

3. Simard, S.W., Mycorrhizal Networks Facilitate Tree Communication, Learning, and Memory, in Memory and Learning in Plants, F. Baluska, M. Gagliano, and G. Witzany, Editors. 2018, Springer International Publishing: Cham. p. 191-213.

4. Baluška, F., S. Mancuso, D. Volkmann, and P. Barlow, The 'root-brain' hypothesis of Charles and Francis Darwin. Plant Signaling \& Behavior, 2009. 4(12): p. 1121-1127.

5. Simard, S.W., D.A. Perry, M.D. Jones, D.D. Myrold, D.M. Durall, and R. Molina, Net transfer of carbon between ectomycorrhizal tree species in the field. Nature, 1997. 388(6642): p. 579-582.

6. Beiler, K.J., D.M. Durall, S.W. Simard, S.A. Maxwell, and A.M. Kretzer, Architecture of the woodwide web: Rhizopogon spp. genets link multiple Douglas-fir cohorts. 2010. 185(2): p. 543-553.

7. Gorzelak, M.A., A.K. Asay, B.J. Pickles, and S.W. Simard, Inter-plant communication through mycorrhizal networks mediates complex adaptive behaviour in plant communities. AoB PLANTS, 2015. 7: p. plv050.

8. Queller, D.C. and J.E. Strassmann, Beyond society: the evolution of organismality. 2009. 364(1533): p. 3143-3155.

9. West, S.A. and E.T. Kiers, Evolution: What Is an Organism? Current Biology, 2009. 19(23): p. R1080-R1082. 